\title{
An Architecture for \\ Multi-User Software Development Environments
}

\author{
Israel Z. Ben-Shaul \\ Gail E. Kaiser \\ George T. Heineman \\ Columbia University \\ Department of Computer Science \\ 500 West 120th Street \\ New York, NY 10027 \\ (212) 854-2736 \\ fax: (212) 666-0140 \\ israel@cs.columbia.edu \\ TR CUCS-012-92
}

May 11, 1992

\begin{abstract}
We present an architecture for multi-user software development environments, covering general, process-centered and rule-based MUSDEs. Our architecture is founded on componentization, with particular concern for the capability to replace the synchronization component to allow experimentation with novel concurrency control mechanisms - with minimal effects on other components while still supporting integration. The architecture has been implemented in the MARVEL SDE.
\end{abstract}

(C)1992 Israel Z. Ben-Shaul, Gail E. Kaiser, and George T. Heineman

subjects: Environment frameworks and architectures, Database support for environments, Process support facilities

The Programming Systems Laboratory is supported by National Science Foundation grants CCR-9106368, CCR-9000930 and CCR-8858029. by grants from AT\&T. BNR, DEC, IBM and SRA, by the New York State Center for Advanced Technology in Computers and Information Systems and by the NSF Engineering Research Center for Telecommunications Research. 


\section{Introduction}

Software Development Environments (SDEs) emerged in an attempt to address the problems associated with developing, maintaining and managing large scale software projects. One of the main issues in SDE research is how to construct environments that are integrated, while at the same time flexible and extensible. Although there have been numerous proposals for cooperative transaction models (see [5] for a survey), little has been achieved regarding flexibility and extensibility of such synchronization mechanisms for multi-user SDEs from the system-architecture point of view. Throughout the paper we refer to this aspect of an SDE as the "multi-user" property.

The architectures of process-centered SDEs include process enaction engines, which enable a programmable approach to defining the behavior of an environment to support a particular software development process [32]. The process enaction engine (and the corresponding process modeling language) must be extended with a notion of concurrency consistency and corresponding synchronization primitives (such as in [41]) to become multi-user environments, where the process as well as the data is shared. Rule Based Development Environments (RBDEs) are a class of processcentered SDEs, in which the process is defined in terms of rules and enaction is achieved through rule chaining. Examples include CLF [34], Oikos [1] and Merlin [45]. Multi-user RBDEs must support synchronization among automated chains of activities as well as activities directly invoked by users. In process-centered SDEs and RBDEs, as well as general SDEs, the architecture must also support interprocess communication, scheduling and context switching, transaction and lock management, and other facilities on which synchronization depends.

This paper presents an architecture for multi-user SDEs (henceforth MUSDEs) that is intended to support the requirements of general, process-centered and rule-based MUSDEs. The emphasis is on identification of the system's components and on the interfaces and interrelations among them rather than on application of specific synchronization policies. We have implemented the architecture for the MARVEL RBDE [28], which was previously a single-user system. This work is complementary but orthogonal to the research done by Barghouti and Kaiser on cooperative transaction management for RBDEs in general and MARVEL in particular [3, 4]. The focus of their work has been on modeling coordination and cooperation, whereas here we focus on the architectural facilities that enable the implementation of such sophisticated synchronization mechanisms.

In section 2, we give the necessary requirements that an MUSDE must fulfill, by definition, and additional desired properties. Section 3 introduces the architecture, its main characteristics and functionality. Section 4 explains the rationale behind the architecture. Section 5 describes the implementation for MARVEL and our experience, including changing and tailoring some of the components. Section 6 compares to related work. Section 7 briefly evaluates the architecture and summarizes our contributions.

\section{Requirements and Desired Properties}

Data-sharing - We distinguish between "product" data and "control" data: the former represents the actual data elements under development (i.e., source files, object files, design documents, etc.), while the latter represents the data used by the SDE to manage the project. Examples of control data for a source file include its version, compilation status, reservation status, etc. Product data may be integrated with control data (e.g., an object is defined as having "control" state attributes 
and file attributes that point to "product" items) or may be maintained separately. In general SDEs, control data represents the status with respect to a hard-coded policy, whereas in process-centered SDEs, control data reflects the state of the specific process in action.

Data-consistency - An MUSDE synchronizes concurrent access to the SDE's data to maintain its consistency, e.g., it prevents data from being garbled by conflicting accesses (such as multiple independent updates) to the same or related data items. Product data can be maintained either by the SDE or in the file system; however, control data must be maintained by the SDE. But access to both must be synchronized, either in a centralized or a distributed fashion, and in the latter case can be tightly integrated within each user's workspace or separate in a DBMS.

Process-sharing and process-consistency - In addition to data-consistency as above, which is required for all MUSDEs, process-centered SDEs must maintain process-consistency, as specified in the process modeling language. In order to maintain process-consistency, the process-engine must maintain a global view of the process. Again, this can be done in either a centralized fashion, or in each user's workspace provided that the necessary information is replicated among users. For example, consider a constraint taken from the "ISPW problem" [18], where a member of group PROGRAMMER cannot make any code changes before some or all members of the Configuration Control Board (CCB) have approved it. The MUSDE must ensure that the constraint is applied to all involved participants (or at least programmers and CCB members).

Whereas the above characteristics are required to exist in MUSDEs of the indicated classes - by definition - the following represent additional properties that are desired in an MUSDE. These properties together form the basis for the rationale behind our architecture.

Perhaps the most important property from the architectural point of view is flexibility in selection and application of synchronization mechanisms. The idea is to be able to replace or modify concurrency control policies, both globally (i.e., for all users of the system) and locally (among selected groups of users). Some proposed concurrency control models, such as transaction groups [20], support this capability to a limited extent in that the policy for each group can be specified in a formalism supplied by the implementation [39]. What we have in mind is more general: The architecture should be constructed such that the entire synchronization component can be replaced with minimal (preferably no) code changes to other parts of the system. This enables to conduct cost-effective expurimentation, which is important in such a novel research area.

The architecture should support synchronization components whose transaction models range from classical atomicity and serializability to long, interactive operations and cooperation. As noted in [8], any synchronization mechanism for an MUSDE must take into account that many activities in software development are long - conventional atomic and serializable transactions are not suitable, and interactive - response time is more important then overall throughput. Cooperation is needed to enable sharing and exchange of information during parallel development.

Extensibility and broad scope of application. An MUSDE should be able to be extended with new tools, including integration of tools not specifically developed for the MUSDE [15].

High Visibility - An MUSDE implemented on a window-based platform should provide users with graphical visualization of at least the control data, and preferably the product data as well. Since SDEs often support complex and highly structured data models, it is especially desirable to be able to display the types and relationships of all objects of the environment. This means that an MUSDE has to maintain up-to-date information as it is dynamically changed by multiple users. 
Recovery - Persistence of product data can be provided by the host file system, but persistence of control data must be provided by the MUSDE. Recovery is an important aspect that ensures consistency of persistent data in case of external and internal failures. We distinguish between concurrency control, which is required by definition, and recovery, which - although not mandatory is a highly desired property for industrial-strength environments. Traditionally, these two functions are both carried out by the "transaction manager".

\section{The Architecture}

\subsection{Overview}

Two major principles underlie the overall design: componentization and layering. According to the componentization principle, a complex system should be built from independent, loosely-coupled and replaceable components. These components must have flexible interfaces and support a variety of different policies potentially employed by alternative interacting components (i.e., components that provide the same services in different ways). Layering is a paradigm in which each component provides services only to the next higher layer and receives services only from lower levels. Layering lowers complexity by reducing inter-component linkages.

Componentization is becoming popular in operating systems (e.g., the replaceable pager in Mach [46]) and databases [44,35], and the layering approach has also been followed in other domains such as communication protocols [13] and databases [9]. The combination seems especially promising for SDE technology, which is by nature subject to changes [42]. We suggest the potential to revise any system component (although with differing degrees of difficulty). Our major concern here is to be able to modify the synchronization mechanism with minimal effects on task management and the remainder of data integration and repository management, which implies that transaction and object management should be separate (as in Camelot [17]).

The generic architecture is depicted in Figure 1, using the terminology of the "toaster" reference model [16]. We concentrate in this section on explaining how things work, and defer to section 4 the discussion on why we chose to design the architecture this way.

The architecture follows the conventional client-server model. Each active environment with a populated objectbase is managed by a single centralized server, and multiple clients are distributed, each representing a user session that lasts from invocation to exit. Both the server and each client are implemented as individual operating system processes. Each client serves as a front-end to the human user and as an activity execution unit. The concept of activity encompasses all operations done to product data, such as editing and testing, via internal or external tools; it does not include operations on control data (although these might occur as side effects). Clients may spawn child processes to execute activities.

The server provides data integration and repository and task management services. Service requests always originate at a client, but most requests that involve access to product and/or control data are sent to the server after client preprocessing. The server validates and processes the request before returning to the client with the desired information and/or instructions to the client to execute a specific activity. The server mediates access to both control and product data, and modifies control data according to the environment specifications.

The architecture distinguishes between normal users and an environment administrator. The ad- 


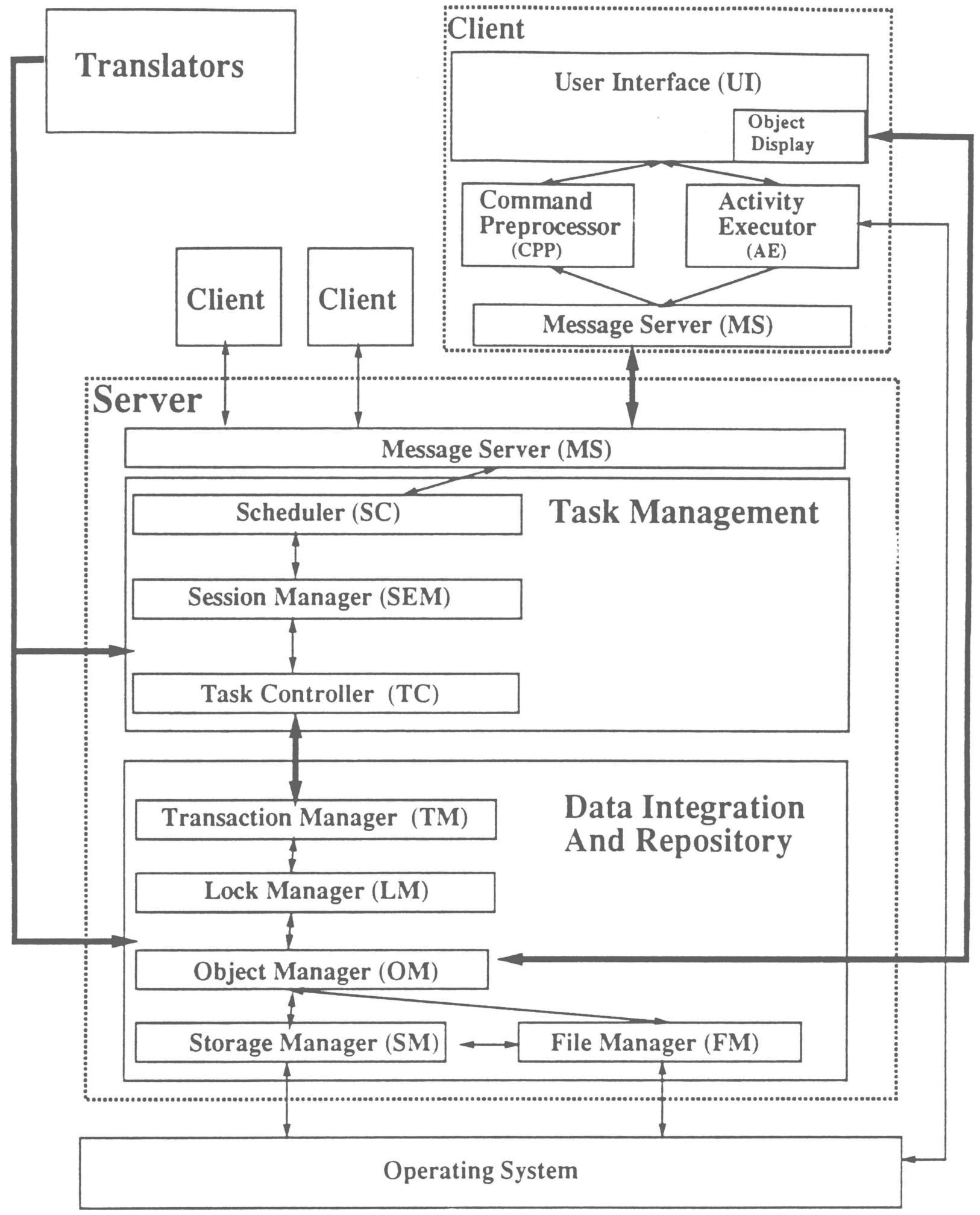

Figure 1: An Architecture for Multi-User SDEs 
ministrator's role resembles that of the Data Base Administrator in DBMSs. The administrator uses a privileged client to define the data model (schema) and any integrity constraints on the data; the process model, if any; and the programmable aspects of the synchronization policy, if any.

\subsection{The Server}

\subsubsection{Task Management}

Translators - Any SDE that allows to define the data model for the control and/or product data must have a data definition translator. In process-centered environments, a process model translator is also needed. MUSDEs with programmable synchronization require yet another translator. Translation can be on-line, in which case this component acts as a loader for other interpretive components, or off-line, in which case it "compiles" the specifications into internal form and is not actively involved at run-time.

Scheduler $(S C)$ - Schedules requests from clients for services, including context-switching. Before a client is serviced, SC makes two contexts active: the client's session-context and the specific task-context (see below).

Session Manager (SEM) - Encapsulates an entire session between a specific client and the server, that is, all requests that occur from invocation to exit of the client. SEM can: (1) maintain the user-specific environment and operating system parameters for general configuration purposes; and (2) store enforcement information that pertains to the entire session (as opposed to task-specific information). For example, users of a specific SDE might explicitly "attach" to a specific subprocess to perform during that session [27].

Task Controller (TC) - This is the central component of the environment, which provides most of the services to the client. A task is defined as any activity initiated by a client together with all the derived operations carried out by the environment, such as automation and enforcement actions. For example, an SDE might have a constraint that when an interface to a function $\mathbf{F}$ is modified, all source files that call $\mathbf{F}$ must be marked for modification. The modification of $\mathbf{F}$ and the marking of dependent files is considered one task. In a general MUSDE, TC may degenerate to a command interpreter, perhaps with a query processor. In process-centered environments, this component includes the process engine, in charge of enacting the process. TC operates in the context of the current session, but maintains a task context for each active task in the system.

\subsubsection{Data Integration and Repository Management}

Transaction Manager (TM) - Maintains the integrity of the data in case of concurrent access and failures. In process-centered MUSDEs, TM also maintains process-consistency. However, it is not responsible for detecting any conflicts due to concurrent access, but only for resolving them.

A "transaction" can map to a single activity or to a single task, but usually not to a session, since this would imply coarse-grained concurrency. There are no specific guidelines for the implementation of concurrency control or recovery, except for the restriction to locking-based mechanisms. For example, an environment may use a "blocking with deadlock resolution" mechanism or a "nonblocking with abort" mechanism, or a combination of both. Also, TM may use flat transactions or nested transactions [30] that model the nesting of activities and subtasks within a task. 
TM-TC interface - The interface between TC and TM is a critical issue as it bridges the border between the task level and the data level. It is desirable for T.M to be independent of any specific task model and for TC to be independent of any specific transaction model, so that either can be replaced with minimal overhead. A predefined set of transaction primitives known to TC must be supported by any TM, with the set flexible enough to support many synchronization policies. However, semantics-based concurrency control [21] inherently requires some knowledge of the task level to resolve conflicts that are context-sensitive. This implies that the TC-TM interface may need to be augmented with a mediator component that reconciles information from both levels.

Lock Manager (LM) - Usually considered a subcomponent of the transaction manager, LM is treated in our architecture as a separate component. Its main role is to detect any potential violations of the data-consistency constraints, as defined by a lock-compatibility matrix. LM must permit a broad range of lock modes to enable TM the freedom to choose those that meet its needs. But the separation of LM, TM and OM makes it impossible to predict what lock set and compatibility will be needed. However, viewing LM merely as a "mechanical" conflict-detector enables it to be table-driven, with the tables loaded during system initialization. This means customizations of TM affect LM only through the tables.

An additional property of LM is to be able to hold multiple locks on objects, on insistence from $T M$, even when they violate the defined compatibility. This is useful for implementation of nonconventional concurrency control policies. For example, transaction groups may allow several transactions in the same group to share transient results. ObServer [25] is a multi-user data server with a rich lock set, including communication modes (for notification), which is capable of supporting transaction groups. This approach provides flexibility in transaction management but is not extensible. In contrast, we regard lock management as a mechanism to detect conflicts only, for an arbitrary set of lock modes; ObServer's communication modes can be implemented in LM with proper support from TM as part of conflict resolution.

Object Manager (OM) - Implements the data model, provides persistence, and performs all requests for access and modification of both control and product data. We assume a generic object-based data model with optional class ("is-a") hierarchy, composition ("is-part-of") hierarchy, and arbitrary relationships between objects ("links"). An object may represent purely control data, an encapsulation of product data, or a combination.

OM-LM interface - For componentization to work, it is important that OM provide the upper layers with an object abstraction that avoids concern with any internal representation. Further, upper layers should not know whether objects are in main or secondary memory [38]. The main difficulty with separating $O M$ and $L M$ is that data-consistency specifications may need to be extended for a specific data model. For example, composite objects and links among objects may require "intention" lock modes for ancestor and linked objects, respectively. This predefined set of lock "extensions" is understood by LM, allowing a wide variety of object-based and entity-relational data models but precluding the possibility of replacing objects/entities with a radically different form such as relations (such a change would also seriously affect the upper layers, notably query processing in TC, impeding componentization).

Storage Manager (SM) - Responsible for low-level disk and buffer management for control data. It deals with untyped, raw data, and interacts with the underlying operating system. If the product data is maintained in ordinary files (as is often the case with SDEs), SM interacts with the File Manager to access "file objects". The separation between storage, object, lock and transaction management distinguishes our architecture from most other systems that provide data management. 
In effect, we take apart ObServer's functionality into SM, LM and TM, and stick in between object management functionality such as found in Mneme [31].

File Manager (FM) - Necessary only when the MUSDE uses file-based tools; otherwise, SM handles product as well as control data. However, a control data object may still encapsulate one or more files as attributes, in which case objects abstract the file system by providing typing and relationship information. FM is responsible for accessing the files requested by the object manager, passing them on to the upper layers and eventually to the clients (for a shared file system such as NFS, only path names need be passed rather than the actual file contents).

Mapping our architecture to the toaster model, data integration and repository management services are in the server, and user interface services are in the clients, as expected. The interesting mapping is that of task management. We divide this between the client and the server, where the client is responsible for "task execution" and the server for the rest.

\subsection{The Client}

User Interface and Objectbase Display Manager (UI) - Provides the human user interface to all environment services, including a display of the entire objectbase (subsets can be viewed via browsing). This feature introduces the challenge of keeping the display up-to-date, since the objectbase is dynamically changed by multiple users, including modifying, adding and deleting objects and/or relationships between objects. A compromise is made, where clients maintain a cache of the structure of the objectbase, mainly for reference purposes in selecting arguments to commands, but not the actual contents of objects. An example is shown in Figure 2.

Activity Executor ( $A E)$ - Interacts with tools in an environment-specific manner. This might include interaction with the operating system for spawning child processes with suitable command lines and transforming data to/from objectbase and tool formats. There may or may not be communication between the $\mathrm{AE}$ and the activity and between the $\mathrm{AE}$ and the server during activity execution.

Command Preprocessor (CPP) - This component is open-ended. It includes formatting of requests for services so that they conform to the interface specifications of the various service providers in the server (fronted by TC), and executes local services that do not affect other users or the software development process. An example of the former is an ad-hoc query parser that performs syntax checking and passes to the server a parsed query. An example of the latter is the "help" facility. CPP has no significant impact on the overall architecture.

Message Server (MS) - Transfers information between the client and the server over the communication medium. An important functionality is the capability to linearize and delinearize the objectbase structure when the image is updated at the client. MS must preserve the object abstraction so that both ends can refer to objects identically.

\subsection{An Example}

Joe, a programmer, wants to change a source file to fix a bug. There are other active users working simultaneously on the same project. He logs into the SDE by invoking a client. The client initiates a connection with the server, passing along session variables. On the server side, SEM initiates a new session context. The client then receives a visual display of the objectbase for the user's screen, and Joe can then start to work. 


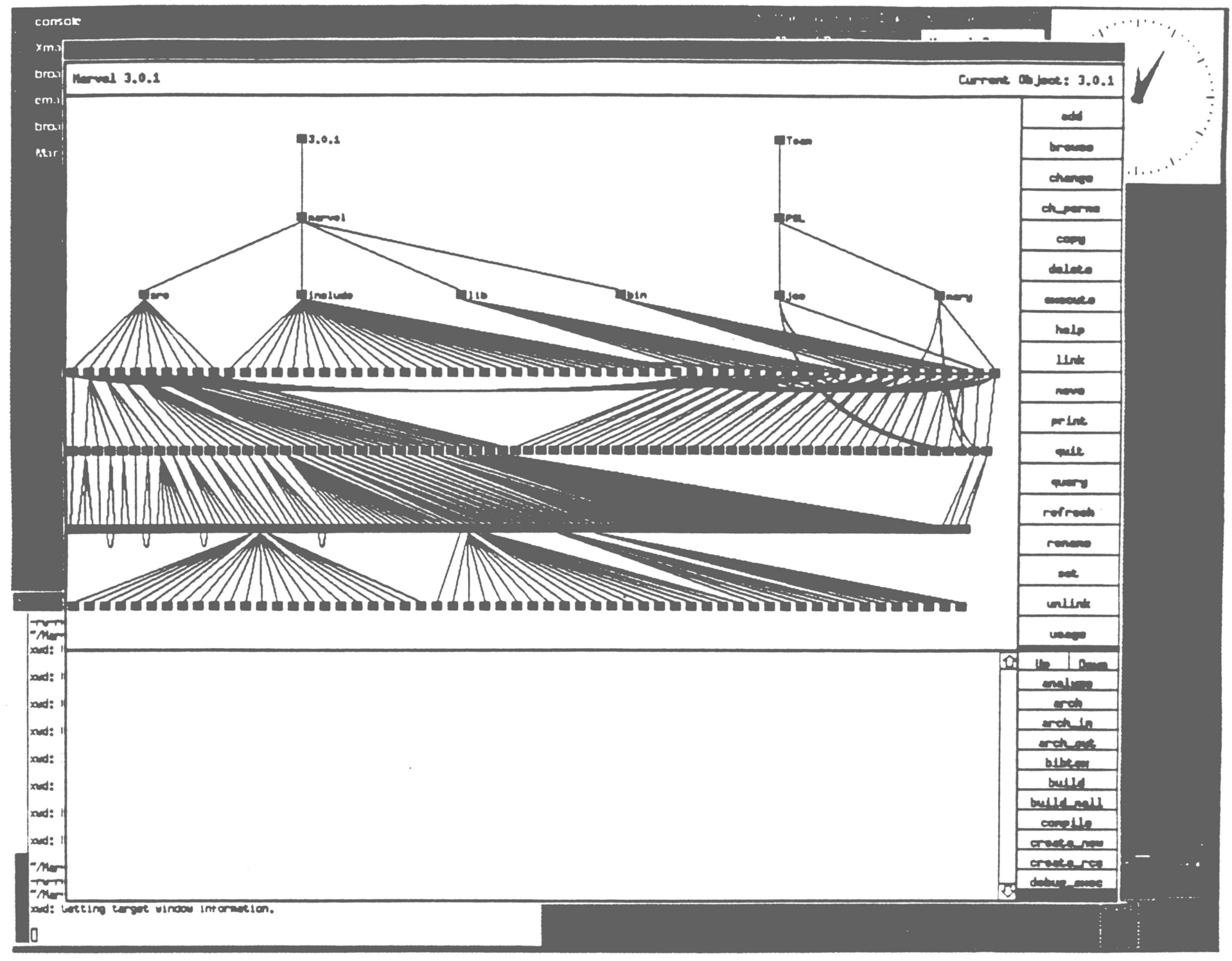

Figure 2: Marvel Objectbase Display 
He proceeds with a request $\mathrm{R} 1$ to edit a file $\mathrm{F} 1$, represented by an object $\mathrm{O} 1$. After preprocessing to check whether $\mathrm{O} 1$ exists and is unambiguous, the client sends the request to the server. When R1 is scheduled, SC updates SEM to point to the appropriate client's session and task contexts, and passes R1 to TC. TC tells TM to begin a transaction, which might be a subtransaction of some in-progress nested transaction when $\mathrm{R} 1$ is part of some on-going task. TC handles any taskrelated constraints or triggers for $\mathrm{R} 1$, and then issues requests to TM to access the objects required, directly by the activity (tool) or indirectly by TC. TM requests the appropriate locks from LM for these objects. LM performs the lock requests by inspecting its previously placed locks and lock tables. If the request is denied due to a conflict detected by LM, TM resolves the conflict (e.g., by aborting or suspending some offending transactions, or by allowing apparently conflicting requests and insisting LM place the locks anyway, depending on the synchronization policy). If the locks are granted, $L M$ then requests $O M$ to access the objects. This may involve interaction with SM and FM. If all goes well, the relevant information propagates back up to this client, and SC selects the next client request.

$\mathrm{AE}$ then executes at the client in an environment-specific manner, for example, by spawning a child process to invoke a tool, pass input from the server, accept output and status code after the tool terminates, and report these to the server. After SC restores the client's contexts, TC handles any task-related constraints or triggers for R1 (i.e., there may be task-related operations both before and after execution of each activity). This mode of interaction continues until there are no more activities to execute or until an exception occurs (e.g., a task is aborted due to intervention of concurrency control or a failure), and the task is terminated. Notice that while a client is executing an activity, the server is not bound in any way to that client and can serve other clients.

\section{Alternatives, Decisions and Justifications}

\subsection{Client-Server Separation}

The first issue to consider is the degree of distribution of the MUSDE. The two obvious alternatives are to fully centralize services or to fully distribute them among clients. In the first case there would still be minimal clients, at least operating system shells, to allow multiple users to communicate with the environment; but all control and product operations would take place in the server. In the second case there would be no dedicated server at all, but only clients, with all control and product operations executed in a client and shared only via communication directly among clients.

We chose a hybrid approach, in which clients are responsible for long duration activities and the server is responsible for relatively short term task control and synchronization. Maintaining dataand process-consistency internal to the server reduces communication overhead, while farming out interactive and/or computation-intensive activities to the relevant clients keeps computation overhead low and response time high. This division of labor seems to best exploit today's high performance workstations and high capacity server machines.

Locating task control in the server does not preclude the possibility of different "views" for different clients [6]: they can be managed by the server as part of the session context. Further, the serverclient separation does not prevent distribution of the server itself into multiple server processes. with communication among themselves to handle decentralized data, process and synchronization. Our intent is to instead make an inherent distinction between the roles of clients and server(s). 


\subsection{Separation of Transaction and Lock Management}

The main reason for decoupling TM and LM is to distinguish conflict-detection from conflictresolution, where the former is a mechanical procedure that reports any violations of the defined consistency and the latter is an elaborate procedure that decides how to resolve a conflict when it arises. This separation enables to modify and/or replace synchronization policies without affecting the underlying conflict detection. Furthermore, the fact that LM has no knowledge of the semantics of the various lock modes (since it does not have to resolve conflicts) enables to implement LM in a way that it can be reconfigured externally via tables, without any code changes. The decoupling of transaction management from lower levels also brings TM closer to task management, enabling semantic-based concurrency-control without concern for low-level data management. This separation contributes perhaps more then anything else to the flexibility of the system with respect to concurrency control. Examples that demonstrate this flexibility are given in section 5 .

\subsection{Tunable Lock Management}

The alternatives are: (1) a non-locking policy, where concurrency control is optimistic (as in NSE [24]); (2) a hard-coded lock set and lock-compatibility matrix; and (3) a dynamic lock set and lock-compatibility matrix. We addressed hard-coding versus externally-defined lock tables in section 3.2. Optimistic concurrency control may be useful when conflicts are known to be rare, provided that the "resolution" is done by "merging" changes of conflicting operations, since rolling back long operations would be unacceptable in most situations. However, an effective merging procedure for source code is still beyond the state of the art (as evidenced by [26]), and sometimes impossible, e.g., there is no general way to merge two versions of a data file created by conflicting operations (although [19] gives some hope of advances). In addition, rolling back interactive operations is generally unacceptable.

\subsection{Objectbase Visibility}

The two obvious alternatives are to keep an entire replica of the objectbase at each client, or to display only those objects that are actually used by a client. Note that in any case control data manipulation is done at the server, so the issue is not where to modify the data, but rather how to display it. Therefore, we can treat anything displayed at the client as a read-only replica. The main problem with keeping an entire replica is that it is very expensive and unnecessary. Objects in a MUSDE can be very large (e.g., large files) and may change frequently, causing tremendous communication overhead. On the other hand, displaying only objects that are used does not fulfill the "high visibility" property.

We chose a hybrid approach, in which the structure of the objectbase is maintained, but not its contents. For each object, we maintain a cache of its name, type, unique ID and relationships to other objects. This is sufficient to be able to view the entire objectbase, while still compact in volume for transmission by MS.

Another consideration is how frequently the display should be updated. The alternatives are to: (1) Broadcast every change to all active clients; (2) Refresh periodically; and (3) Refresh "on-demand". as determined by the server. The third alternative is preferred as it saves communication overhead while keeping information reasonably up to date. This is easily implemented by maintaining a 
timestamp in the client's session-context and "piggybacking" a refreshed objectbase image on the response to each client's next request. An explicit "refresh" command is also provided for the user who wants to ensure his/her image is up-to-date.

\section{Implementation for Marvel}

The MARVEL 3.x architecture is illustrated in Figure 3. It can be viewed as a rule-based instance of the generic MUSDE architecture of Figure 1. The client structure is essentially the same. The server reflects TC in three sub-components: query processor (QP), command processor for built-in commands (CP), and rule processor (RP) responsible for process enaction. It also adds a Coordination Manager (CM) as a mediator between TM and TC. MARVEL's translation and loading component is collectively called Loader. Tool envelopes and data, process and coordination models are written (by the administrator) in various notations and loaded (again by the administrator) using a privileged client, tailoring the environment's behavior according to these specifications. The MARVEL daemon, not shown, automatically starts a server on the appropriate objectbase when its first client logs in, and kills the server after the last client has exited.

\subsection{Process Modeling and Enaction}

The process is defined in terms of rules, each representing a single activity. Each rule consists of a name; a list of typed parameters; a condition that represents the properties that must hold on actual parameters and other objects bound in the condition for the rule to fire; an activity that specifies a "product" activity and its arguments; and a set of mutually exclusive effects consisting of assertions to the objectbase that reflect the result of executing the activity. Rules are implicitly related to each other through a match between a predicate in the condition of one rule and an assertion in the effect of another rule.

Process enaction in RP is done through chaining. When an activity is requested, the condition of the corresponding rule is evaluated. If not satisfied, RP attempts to satisfy it by backward chaining to other rules whose effects may satisfy the user-invoked rule. This is done recursively, until the condition is satisfied or all possibilities are exhausted, in which case the activity cannot be executed. When the activity returns from the client (assuming the rule's condition was satisfied), RP asserts the effect indicated by the status code returned from $A E$ and then recursively forwards chains to all rules whose conditions have become satisfied. MARVEL distinguishes between consistency and automation chains, which are specified by annotations on condition predicates and effect assertions in the rules. Consistency chains propagate changes and are by definition mandatory and atomic. Automation chains automate activities and are by definition optional; they may be terminated at any point or "turned off" entirely. The details are outside the scope of this paper; see [6].

\subsection{Task Management}

MARVEL's scheduler implements a simple non-preemptive scheduling policy (FCFS). However, Nonpreemptive scheduling does not imply that an entire session, or even an entire task, is handled by the server atomically. Instead, we exploit the natural "breaks" within and among tasks, at which point the server performs context switch and turns to the next client request. That request might 


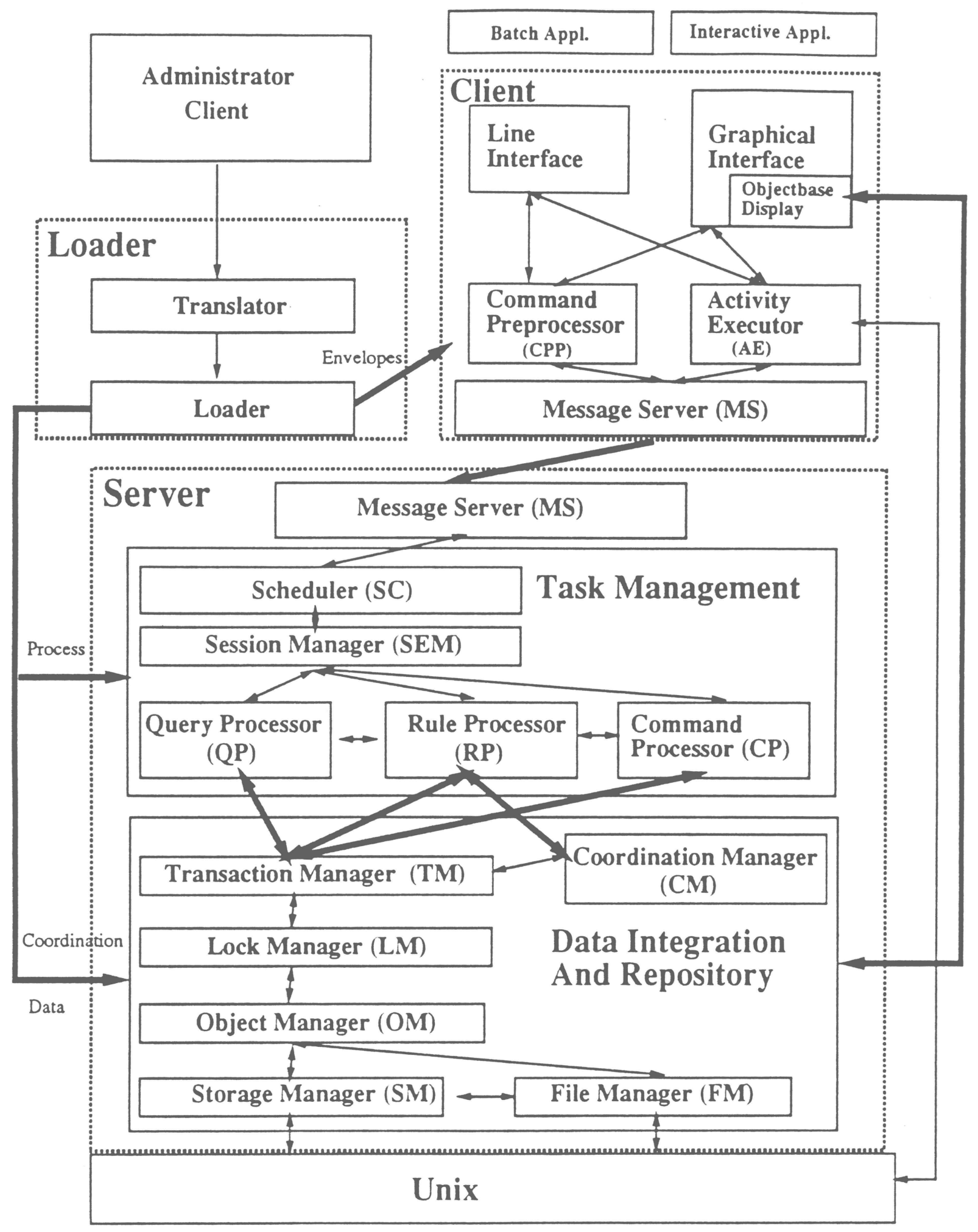

Figure 3: MaRvel 3.x Architecture 
resume an in-progress task or initiate a new task.

$\mathrm{RP}$ is the heart of task management. A task consists of all rules executed during backward chaining, followed by the user-invoked rule (which caused the backward chain), followed by all rules executed during forward chaining. RP operates in a specific task context, consisting of information necessary for maintaining the state of the task. The main data structure is the rule stack, one per task. Since backward chaining is multiply-recursive and generates an AND/OR tree, (i.e., in some cases a rule's condition may be satisfiable only by application of a set of rules, and in other cases by any one of many possible rules), the rule stack is implemented as a multi-level stack, where each level consists of an ordered set of rules that correspond only to the first rule in the previous level, and are not related to other rules in the previous level. The same stack is used for forward chaining, although here a standard stack mechanism is sufficient.

One problem of multi-tasking rule processing is that multiple instances of the same rule may be fired concurrently by the same or different clients, and since they all fire in the context of one RP (i.e., one address space), rules cannot contain any private data. This problem is solved by making rules reentrant. Each invocation entails creation of a rule-frame, which consists of a pointer to the (read-only) rule and a dynamically allocated data section, which it retains throughout the entire life cycle of a rule chain.

\subsection{Data Integration and Repository Management}

TM supports a nested transaction model in which a task is modeled as a top-level transaction, each consistency chain is a subtransaction consisting of a further level of subtransactions corresponding to individual rules, whereas each rule in an automation chain is an independent subtransaction on its own. By definition, an entire consistency chain is executed to completion or rolled back as if it never started, while the latest rule in an automation chain can be aborted without affecting the rest of the chain. In MARVEL 3.1, CM will serve as a mediator between data and task management; $\mathrm{CM} / \mathrm{RP}$ and CM/TM interfaces have already been partially implemented.

MARVEL's composition hierarchy is based on ORION [29], using intention locks for ancestors. When object $\mathrm{O} 1$ is locked, all $\mathrm{O} 1$ 's ancestors are locked in the corresponding intention mode. Intention locks are generally weaker than the corresponding descendant locks, and their goal is to protect objects from being affected by an operation on an ancestor. For example, when object D is locked in $\mathrm{L}$ mode, IL locks are placed on all its ancestor, where IL is compatible with any operation that would not affect $\mathrm{D}$. In particular, it is compatible with another IL lock. This idea can be extended to linked objects as well as ancestors, but this is not supported in MARVEL.

LM reads three tables when initialized: compatibility matrix, ancestor table and power matrix. The compatibility matrix defines the set of lock modes and the compatibility of any two locks modes. The ancestor table indicates which lock to apply to the ancestors of the object being locked in a certain mode. The power matrix determines which lock has precedence given two locks requested by the same transaction.

SM uses the Unix dbm package. Although more sophisticated data management strategies can be supported by dbm, SM loads the entire objectbase (but not files objects) into memory at server startup. FM is implemented by a collection of system calls that map the object name-space to the file system name-space, and perform operations on a "hidden" file system rooted at a directory representing a populated objectbase of an environment. MS uses Internet sockets. 


\subsection{MaRVEL Client and Loader}

UI includes both graphical and command line interfaces with the former providing full objectbase browsing capability (conceptually communicating with $\mathrm{OM}$ directly) and the latter supporting batch processing scripts as well as dumb terminals. CPP includes an ad-hoc query parser (available now in 3.0 .1 but not in 3.0). AE is the most complex component of the client. It is in charge of spawning child processes for executing envelopes, basically shell scripts, for the various tools defined in the environment. AE communicates with envelopes through pipes in a "black-box" fashion: inputs are provided at the beginning of activity execution, and output and a status code are collected at the end. The details are beyond the scope of this paper; see [22].

The Loader generates a static rule network from the process model, which is used at runtime to determine chaining. This network is loaded into RP, to define process-consistency. The data and process models are tied in the sense that rule parameters and local bindings in the condition of rules are typed according to classes. The data model is used by OM and QP. The various lock tables (a degenerate coordination model) are loaded into LM, to specify data-consistency. A full coordination model using the Control Rule Language syntax described by Barghouti [3] can already be loaded, but was not included in the current release.

\subsection{Experience}

We started with the standard shared and exclusive locks, and intention locks (see Figure 4-a), but then modified the lock tables several times. The final change added two new lock modes and removed one, and changed the compatibility of old modes. The purpose was to provide semanticsbased locking by distinguishing between operations that affect only a single object (e.g., write on a simple attribute) and operations that might affect related objects (e.g., the delete operation removes an object and all its children). Strong Exclusive (SX) and Strong Shared (SS) locks were added, and $X$ and $S$ became compatible with any intention lock (see Figure 4-b). The ancestor table was modified to include intention locks for the new modes. This required no code changes to LM and only minor changes to TM to replace requests for locks according to the new semantics. Even these changes would not have been needed for MARVEL 3.1, where the specific lock modes to use for particular arguments of activities and rules will be specified as part of the coordination model. Thus, a dramatic change in conflict detection can be achieved with very small overhead.

We also started with a flat transaction model, in which an entire chain executed as a single transaction. This made it impossible to treat different subsets of a task differently. For example, we could not abort an automation subchain without rolling back consistency subchains descended from the same user-invoked rule. We replaced TM with nested transactions. Each rule triggered during an automation chain, together with any consistency subchains emanating from it, is a subtransaction that can be aborted without affecting the top-level transaction or other subtransactions. Again, this major change had no impact whatsoever on LM, and required only trivial changes to RP.

CM is being developed to support programmable conflict resolution. In MARVEL 3.1, the administrator will be able to define an optional set of control rules to specify scenarios when the default policy above may be relaxed, and prescribe appropriate actions in each such case. We have already built a facility whereby $\mathrm{CM}$ accesses RP's rule stacks, since control rule scenarios require inspection of conflicting rule chains. TM already requests CM to try to match its control rules, before proceeding to the default conflict resolution policy, and C.M can select the closest match of a set of 


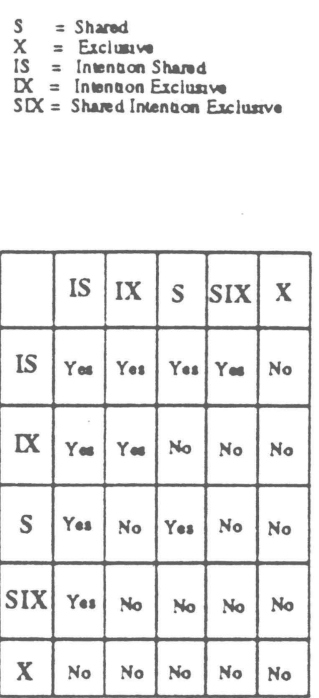

4-a
$S=$ Shared

$X=$ Excluive
IS $=$ Intention Shared

$X=$ Internoon Exaiviou

$S S=$ Strong Shared

$S \mathrm{X}=$ Strong Euclaurve

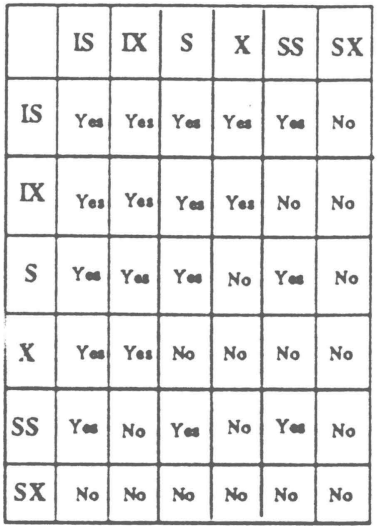

4-b

Figure 4: Initial and Revised Compatibility Matrices

control rules. But we are still investigating the desirable operations for the actions, so the semantics of a non-empty control rule base are undefined. We will have to add to TM a "marking" phase, to annotate objects left temporarily inconsistent by control rule actions that suspend or terminate in-progress consistency chains, and an "unmarking" phase to attempt to restore consistency when access to such objects is requested. We anticipate no other changes to TM, small if any to RP, and none to other components.

The current release provides only one active task per client, but MARVEL 3.1 will allow manual switching among multiple in-progress tasks within a single client. This requires substantial changes to $A E$, and some to CPP, but only very minor changes to the server, mainly in the MS component (much of the purpose is to support the actions of the new CM, e.g., to suspend or abort in-progress activities). AE has already been enhanced, for release in 3.1, to add "gray-box" integration for extensible tools. This interface allows repeated input and output while an activity is in progress. For example, a multi-buffer editor (such as emacs) makes it feasible for the user to request multiple files for editing incrementally, but our current "black-box" approach requires all inputs/outputs to be requested up front. Again, while major new functionality was added to the client, very minor changes were required in the server.

\subsection{Status}

Marvel 3.x is implemented in C and runs on Sun4s (SunOS 4.1.1) and DecStations (Ultrix 4.2), using X11R4 windows. User, administrator and implementor manuals are provided. MARVEL 3.0 was released for educational institutions and industrial sponsors in November 1991. It included all the features presented in previous sections, except where noted, and has been licensed to ten external sites. "In-progress" variants had been previously demonstrated at several conferences, and in one case released to sponsors. Version 3.0.1, available for licensing since March 1992, is the first version to be fully developed and maintained using C/MARVEL, a MARVEL process for $\mathrm{C}$ development: the source code is available only in a MARVEL objectbase. We are also using 
C/Marvel to develop Marvel 3.1, which is planned for release in September 1992. In addition to the enhancements explained above, this will include: A new user interface based on XView; automatic evolution of existing objectbases for additions of attributes and to the class hierarchy; and the replacement of built-in commands, currently supported by the command processor, by built-in operations executable from rules, which will make the system fully rule-based.

\section{Related Work}

SMS (aka Gypsy) $[12,37]$ is basically an extension of version control-based systems (e.g., RCS, SCCS [36]) tightly integrated with an extended operating system. Synchronization is manual, and users work in isolation, each in his/her own "workspace". Although SMS provides a mechanism for multiple users to access data objects concurrently by specifying a list of users that can attach to a workspace, it provides no means for coordinating their access.

NSE [24] supports parallel and distributed development through an optimistic concurrency control mechanism, which allows multiple users to access objects concurrently without locking them. When multiple users modify the same file, a merge process aids in merging the multiple versions. File management is based on the concept of environment, a separate workspace with its own copy of the file system. Environments can be arbitrarily nested. This hard-coded policy dictates a methodology for software development, in which developers work primarily in isolation and conflicts are rare.

Arcadia [43, 33] is a process-programming environment based on the extensive research in SDE technology underway by the Arcadia consortium. Like our architecture, it is constructed out of layered components that are intended to be replaceable. However, although process-consistency from the process-programming point of view is addressed extensively by Sutton [40], an independent synchronization component is conspicuously absent from the architecture. We guess that multi-user synchronization is provided by the object management system.

Melmac [14] is a process-centered environment that distinguishes between application-level and intermediate-level representations of the process. Unlike our architecture, Melmac's server is primarily concerned with data management and provides a simple transaction mechanism, whereas the clients are responsible for process enaction. One shortcoming evidenced by the examples given in [23] is that since process management is detached from the server, it seems that rule chains cannot be interleaved even during activity execution, which might degrade response time significantly.

Oikos [1] is a rule-based MUSDE that supports concurrency using a hierarchy of blackboards that resemble Linda's tuple spaces [10]. Oikos enables to specify a wide range of services as part of process enactment, including database schemas and transactions. However, while concurrency is an inherent aspect in the Oikos architecture, concurrency control is not, and it is not clear what range of synchronization policies can be supported, nor is it clear how these might be supported.

CLF is a rule-based MUSDE that distinguishes between consistency and automation, but through separate classes of rules rather than annotations on rule predicates as in MARVEL. CLF employs a form of optimistic concurrency control based on merging, with consistency tolerated by automatically placing guards on inconsistent data [2], similar to our notion of "marking". Changes are grouped into evolution steps, which can be undone or redone [11].

Merlin is the closest system to Marvel. From the process modeling viewpoint, the main difference may be that Merlin distinguishes forward and backward chaining styles of rules while MARVEL has 
a single rule base and a symmetric chaining model. There are substantial architectural differences, however: Merlin employs a simple checkin/checkout model, using an object's state as determined by the rules as a lock; there is no support for multiple locking modes; and the objectbase display is limited to each user's working context (although there is a refresh mechanism). It appears that chaining operates in each user's working context (client) as opposed to a centralized server.

\section{Evaluation and Contributions}

The drawbacks of our architecture include:

- Semantics-based concurrency control and componentization are, in some sense, conflicting goals: how can the transaction manager be semantics-based when the semantics are hidden in the task controller? For example, in our work towards MARVEL 3.1's programmable concurrency control, we had to develop a richer interface between the rule processor and the coordination manager than was previously needed for the transaction manager. It seems unlikely that a sufficiently rich general interface - without a sophisticated mediator - can be developed between the task controller and the transaction manager to allow replacement of either without affecting the other.

- Our architecture provides no direct interface between clients and the synchronization components. However, users will need to place explicit requests for notification, if not other purposes; such an enhancement is being contemplated for MARVEL 3.1, where available commands can be defined in the coordination model. We anticipate changes would be required for the command preprocessor as well as the coordination and/or transaction managers.

But there are many advantages of our architecture. At the user interface level, the structural display facility provides for high visibility without the overhead of maintaining complete replicas at the clients. At the task management level, the separation between activity execution and task control provides for process sharing while enabling local execution of tools.

At the data management level, we have made several architectural decisions we believe are unique as well as fruitful: (1) The separation between transaction and lock management allows definition and monitoring of data-consistency independent of the synchronization policy, with minimal overhead. Moreover, this enables to implement sophisticated coordination models, with little effect on other components. (2) A table-driven lock manager allows to modify data-consistency policies with no code changes. (3) The decision to separate transaction management from object management emphasizes our view of support for advanced synchronization models. Essentially, we moved synchronization away from low-level data integration and closer to the semantic, task level. We do not know of any other MUSDE with such functionality.

However, the single centralized server does not scale up to very large numbers of clients. As more clients are added and the objectbase grows, the likelihood of noticeable waits increases. In the short term, improvements can be made by incremental refresh of the objectbase image and preemptive scheduling of tasks. For the longer term, we have sketched a proposal for distributing the server into a hierarchy of local servers, each attached to a group of closely cooperating clients [7]. We anticipate that the clients will not change much and there will be few changes to the basic structure of the server architecture, although several of the components will have to be replaced. 


\section{Acknowledgments}

We would like to thank Naser Barghouti for his numerous contributions to the MARVEL project in general and to concurrency control in particular; Bill Riddle, Brian Nejmeh and Steve Gaede helped shape the functionality of multi-user Marvel; Mark Gisi, John Hinsdale, Tim Jones, Will Marrero, Moshe Shapiro and Mike Sokolsky participated in parts of the implementation effort; Hideyuki Miki, Steve Popovich and students in the E6123 Programming Environments and Software Tools course helped by testing MARVEL as users.

\section{References}

[1] V. Ambriola, P. Ciancarini, and C. Montangero. Software process enactment in Oikos. In Richard N. Taylor, editor, SIGPLAN '90 \& th ACM SIGSOFT Symposium on Software Development Environments, pages 183-192, Irvine CA, December 1990. Special issue of SIGSOFT Software Engineering Notes, 15(6), December 1990.

[2] Robert Balzer. Tolerating inconsistency. In 19th International Conference on Software Engineering, pages 158-165, Austin TX, May 1991.

[3] Naser S. Barghouti. Concurrency Control in Rule-Based Software Development Environments. PhD thesis, Columbia University, February 1992. CUCS-001-92.

[4] Naser S. Barghouti and Gail E. Kaiser. Modeling concurrency in rule-based development environments. IEEE Expert, 5(6):15-27, December 1990.

[5] Naser S. Barghouti and Gail E. Kaiser. Concurrency control in advanced database applications. AC.M Computing Surveys, 23(3):269-317, September 1991.

[6] Naser S. Barghouti and Gail E. Kaiser. Scaling up rule-based development environments. In A. van Lamsweerde and A. Fugetta, editors, 3rd European Software Engineering Conference, volume 550 of Lecture Notes in Computer Science, pages 380-395, Milano, Italy, October 1991. Springer-Verlag.

[7] Israel Z. Ben-Shaul. An object management system for multi-user programming environments. Master's thesis, Columbia University, Department of Computer Science, April 1991. CUCS-010-91.

[8] Philip A. Bernstein. Database system support for software engineering. In 9th International Conference on Software Engineering, pages 166-178, Monterey CA, March 1987.

[9] Alfred Brown and John Rosenberg. Persistent object stores: An implementation technique. In Alan Dearle, Gail M. Shaw, and Stanley B. Zdonik, editors, Implementing Persistent Object Bases: Principles and Practice, pages 199-212, Martha's Vineyard MA, September 1990. The Fourth International Workshop on Persistent Object Systems, Morgan Kaufmann.

[10] Nicholas Carriero and David Gelernter. Linda in context. Communications of the ACM, 32(4):444-458, April 1989.

[11] Don Cohen and K. Narayanaswamy. A logical framework for cooperative software development. In Takuya Katayama, editor, 6th International Software Process Workshop, Hakodate, Japan, October 1990. IEEE Computer Society Press.

[12] Ellis S. Cohen, Dilip A. Soni, Raimund Gluecker, William M. Hasling, Robert W. Schwanke, and Michael E. Wagner. Version management in Gypsy. In Peter Henderson, editor, $A C M$ SIGSOFT/SIGPLAN Software Engineering Symposium on Practical Software Development Environments. pages 201-215, Boston MA, November 1988. ACM Press. Special issues of Software Engineering Notes. 13(5), November 1988 and SIGPLAN Notices, 24(2), February 1989.

[13] J. D. Day and H. Zimmermann. The OSI reference model. In IEEE, volume 71, pages 1334-1340. December 1983. 
[14] Wolfgang Deiters and Volker Gruhn. Managing software processes in the environment MELMAC. In Richard N. Taylor, editor, SIGPLAN '90 4th ACM SIGSOFT Symposium on Software Development Environments, pages 193-205, Irvine CA, December 1990. ACM Press. Special issue of Software Engineering Notes, 15(6), December 1990.

[15] Mark Dowson. Istar - an integrated project support environment. In ACM SIGSOFT/SIGPLAN Software Engineering Symposium on Practical Software Development Environments, pages 27-33, Palo Alto, CA, December 1986. Special issue of SIGPLAN Notices, 22(1), January 1987.

[16] Anthony Earl. Principles of a reference model for computer aided software engineering environments. In Fred Long, editor, Software Engineering Environments International Workshop on Environments, volume 467 of Lecture Notes in Computer Science, pages 115-129, Chinon, France, September 1989. Springer-Verlag.

[17] Jeffrey L. Eppinger, Lily B. Mummert, and Alfred Z. Spector, editors. Camelot and Avalon A Distributed Transaction Facility. Morgan Kaufman, San Mateo CA, 1991.

[18] Marc I. Kellner et al. Software process modeling example problem. In Takuya Katayama, editor, 6th International Software Process Workshop, Hakodate, Hokkaido, Japan, October 1990. IEEE Computer Society Press. In press.

[19] Martin Hardwick et al. Change processing tools for concurrent engineering. Technical Report 91-5, Rensselaer Design Research Center, Rensselaer Polytechnic Institute, February 1991.

[20] Mary F. Fernandez and Stanley B. Zdonik. Transaction groups: A model for controlling cooperative work. In 3rd International Workshop on Persistent Object Systems: Their Design, Implementation and Use, pages 128-138, Queensland, Australia, January 1989.

[21] Hector Garcia-Molina. Using semantic knowledge for transaction processing in a distributed database. ACM Transactions on Database Systems, 8(2):186-213, June 1983.

[22] Mark A. Gisi and Gail E. Kaiser. Extending a tool integration language. In 1st International Conference on the Software Process, pages 218-227, Redondo Beach CA, October 1991.

[23] Volker Gruhn. Validation and Verification of Software Process Models. PhD thesis, Forschungsberichte des Fachbereichs Informatik der Universität Dortmund, 1991. Bericht Nr. 394/91.

[24] M. Honda. Support for parallel development in the sun network software environment. In 2nd International Workshop on Computer-Aided Software Engineering, pages 5-5-5-7, 1988.

[25] Mark F. Hornick and Stanley B. Zdonik. A shared, segmented memory system for an object-oriented database. ACM Transactions on Office Automation Systems, 5(1):70-95, January 1987.

[26] Susan Horwitz, Jan Prins, and Thomas Reps. Integrating noninterfering versions of programs. ACM Transactions on Programming Languages and Systems, 11(3):345-387, July 1989.

[27] Gail E. Kaiser, Israel Z. Ben-Shaul, and Steven S. Popovich. Implementing activity structures process modeling on top of the marvel environment kernel. Technical Report CUCS-027-91, Columbia University, September 1991.

[28] Gail E. Kaiser, Peter H. Feiler, and Steven S. Popovich. Intelligent assistance for software development and maintenance. IEEE Software, 5(3):40-49, May 1988.

[29] Won Kim, Jorge F. Garza, Nathaniel Ballou, and Darrel Woelk. Architecture of the ORION nextgeneration database system. IEEE Transactions on K'nowledge and Data Engineering, 2(1):109-124, March 1990.

[30] J. Eliot B. Moss. Nested Transactions: An Approach to Reliable Distributed Computing. Information Systems. The MIT Press, Cambridge MA, 1985. PhD Thesis, MIT LCS TR-260, April 1981.

[31] J. Eliot B. Moss and Steven Sinofsky. Managing persistent data with Mneme: Designing a reliable, shared object interface. In Advances in Object-Oriented Database Systems, volume 334 of Lecture Notes in Computer Science, pages 298-316. Springer-Verlag, September 1988. 
[32] Leon Osterweil. Software processes are software too. In 9th International Conference on Software Engineering, pages 1-13, Monterey CA, March 1987. IEEE Computer Society.

[33] Leon J. Osterweil and Richard N. Taylor. The architecture of the Arcadia-1 process centered software environment. In Takuya Katayama, editor, 6th International Software Process Workshop, Hakodate, Japan, October 1990. IEEE Computer Society Press.

[34] CLF Project. CLF Manual. USC Information Sciences Institute, January 1988.

[35] Joel E. Richardson and Michael J. Carey. Programming constructs for database system implementation in exodus. In Umeshwar Dayal and Irv Traiger, editors, SIGMOD International Conference on the Management of Data, pages 208-219, San Francisco, CA, May 1987. ACM Press.

[36] M. J. Rochkind. The source code control system. IEEE Transactions on Software Engineering, SE1:364-370, 1975.

[37] R.W. Schwanke, E.S. Cohen, R. Gluecker, W.M. Hasling, D.A. Soni, and M.E. Wagner. Configuration management in biin sms. In 11th International Conference on Software Engineering, pages 383-393, Pittsburgh PA, May 1989.

[38] Eugene Shekita and Michael Zwilling. Cricket: A mapped, persistent object store. In Alan Dearle, Gail M. Shaw, and Stanley B. Zdonik, editors, Implementing Persistent Object Bases: Principles and Practice, pages 89-102, Martha's Vineyard MA, September 1990. The Fourth International Workshop on Persistent Object Systems, Morgan Kaufmann.

[39] Andrea Helen Skarra. A Model of Concurrency Control for Cooperating Transactions. PhD thesis, Brown University, May 1991.

[40] Stanley M. Suttion, Jr. APPL/A: A Prototype Language for Software-Process Programming. PhD thesis, University of Colorado, 1990.

[41] Stanley M. Sutton, Jr. A flexible consistency model for persistent data in software-process programming languages. In Alan Dearle, Gail Shaw, and Stan Zdonik, editors, Implementing Persistent Object Bases Principles and Practice: The \&th International Workshop on Persistent Object Systems, pages 297-310, Martha's Vineyard MA, September 1990. Morgan Kaufmann.

[42] Stanley M. Sutton, Jr., Dennis Heimbigner, and Leon J. Osterweil. Language constructs for managing change in process-centered environinents. In Richard N. Taylor, editor, SIGSOFT' 90 \&th ACM SIGSOFT Symposium on Software Development Environments, pages 206-217, Irvine CA, December 1990. ACM Press. Special issue of Software Engineering Notes, 15(6), December 1990.

[43] Richard N. Taylor, Richard W. Selby, Michal Young, Frank C. Belz, Lori A. Clarke, Jack C. Wileden, Leon Osterweil, and Alex L. Wolf. Foundations for the Arcadia environment architecture. In Peter Henderson, editor, ACM SIGSOFT/SIGPLAN Software Engineering Symposium on Practical Software Development Environments, pages 1-13, Boston MA, November 1988. ACM Press. Special issues of Software Engineering Notes, 13(5), November 1988 and SIGPLAN Notices, 24(2), February 1989.

[44] David Wells. DARPA open object-oriented database preliminary architecture specification, September 1991.

[45] Wilhelm Schäfer and Burkhard Peuschel and Stefan Wolf. A knowledge-based software development environment supporting cooperative work. International Journal on Software Engineering \& Knowledge Engineering, March 1992. In press.

[46] Michael Young, Avadis Tevanian Jr., Richard Rashid, David Golub, Jeffrey Eppinger, Jonathan Chew, William Bolosky, David Black, and Robert Baron. The duality of memory and communication in the implementation of a multiprocessor operating system. In 11th Symposium on Operating Systems Principles, November 1987. 